\title{
Kinetics of human male pronuclear development in a heterologous ICSI model
}

\author{
Estella L. Jones • Olga Mudrak • Andrei O. Zalensky
}

Received: 16 January 2010 / Accepted: 19 February 2010 / Published online: 11 March 2010

(C) Springer Science+Business Media, LLC 2010

\begin{abstract}
Purpose To evaluate human sperm nuclear chromatin decondensation in a heterologous ICSI system using hamster ova injected with human sperm.

Materials and methods Frozen hamster oocytes were injected with Triton X-100 treated sperm and fixed at different time points post ICSI. Oocytes injected with nontreated sperm served as controls. Male pronuclear decondensation was evaluated after staining with DAPI.

Results Sperm cells with partially destroyed membranes and depletion of the acrosome decondense more rapidly and to a greater extent than membrane/acrosome intact cells. Marked variability in pronuclear size was observed for any time point post ICSI, which most probably reflects the heterogeneity in the mature human sperm population.

Conclusion Remodeling of male gamete nuclei in this heterologous ICSI mimics events that occur during natural fertilization in humans and therefore this approach may be used for studies of human sperm chromosomes transformations.
\end{abstract}

Keywords Acrosome Chromatin · Decondensation · Heterologous ICSI · Pronucleus

Capsule Human-hamster heterologous ICSI mimics sperm nuclei transformations that occur during natural fertilization in humans.

E. L. Jones · O. Mudrak · A. O. Zalensky $(\bowtie)$

The Jones Institute for Reproductive Medicine, Eastern Virginia Medical School,

Norfolk, VA 23507, USA

e-mail: zalensao@evms.edu

O. Mudrak

Institute of Cytology, Russian Academy of Sciences,

St. Petersburg 194064, Russia

\section{Introduction}

Fertilization converts two terminally differentiated cells into a totipotent zygote that can form all the cell types in the body. Human eggs, like those of most mammals, are fertilized at meiotic metaphase II. Fertilization activates the oocyte and triggers the completion of meiosis so that the inactive sperm nucleus is, in a coordinated fashion, transformed into a functional male pronucleus (mPN) within the egg cytoplasm. This process consists of complex overlapping stages [1-3]: (1) removal of the sperm nuclear envelope and disassembly of nuclear lamina; (2) substitution of protamines with histones that leads to initial chromatin decondensation followed by recondensation; (3) rebuilding of a nuclear envelope; (4) swelling of the mPN and its migration towards the female PN; and (5) the rapid demethylation of sperm DNA during PN development [4]. And finally paternal and maternal genomes unite and prepare for cleavage forming the mitotic spindle.

Chromosome organization in somatic cells is regarded as an important factor in gene expression. In addition to DNA sequence and histone code, it is an integrated part of the epigenetic mechanisms, which play a major role in fertilization and early embryonic development. Nonetheless, chromosome topology in gametes and zygotes is poorly studied. Recent studies clearly demonstrated that chromosomes in mature human spermatozoa have nonrandom, well-defined, and unique spatial organization [5-7]. In addition, sperm chromosomes have a preferred intracellular positioning [8-10].

Due to the inability to conduct studies with human embryos, adequate model systems are necessary to study sperm chromosome transformation during the early events of fertilization. Recently, intracytoplasmic sperm injection (ICSI) of human sperm into mouse oocytes has been used 
to study histone code during PN development [11]. Here we use ICSI of human sperm into hamster oocytes.

Zona-free hamster oocytes are unique in that they can be penetrated by every species of sperm tested thus far. The ability of human sperm to form pronuclei within zona-free hamster oocytes, the "hamster test" or hamster oocyte sperm penetration assay [12-15] has been used by IVF clinics for years. This test is generally used for the evaluation of numerical and structural abnormalities in sperm chromosomes [16]. It may be also applied for studies of sperm chromatin decondensation. In more recent modification, a single human sperm is injected into each oocyte using the ICSI technique, which eliminates any sperm bound to oocyte surface [17]. After injection, human sperm undergoes decondensation that, at first glance, mimics the formation of pronucleus. ICSI of enucleated mouse oocytes has also been shown to yield a reliable method for chromosome analysis of human sperm [18]. Analysis of sperm chromatin remodeling and behavior of chromosomes during this process has not been performed so far.

In ICSI, capacitation, acrosome reaction and membrane fusion are bypassed. Sperm chromatin enters the ooplasm along with the perinuclear material, acrosome and cell membrane. Eventually, these components disintegrate inside the oocyte. It has been suggested that they may interfere with sperm chromatin remodeling [19-22]. Regardless, live offspring have been born using ICSI in a variety of species, including humans and mice [23]. However, existing reports on higher incidence of chromosome aberrations [24] and lower developmental potentials $[25,26]$ in embryos produced by ICSI indicate that further evaluations on the effects of the incorporation of the acrosome into the oocyte are needed.

In the present study, we explored male pronuclei development with interspecies ICSI utilizing hamster oocytes (HO) injected with human sperm. We established the detailed kinetics of $\mathrm{mPN}$ decondensation using acrosome intact (AI) and acrosome depleted (AD) sperm. We further demonstrated the heterogeneity in $\mathrm{mPN}$ decondensation possibly reflecting intrinsic heterogeneity in the sperm population.

\section{Materials and methods}

\section{Preparation of sperm for ICSI}

Semen samples were obtained from three normozoospermic [27] healthy men (not of proven fertility) enrolled in the donor sperm program at The Jones Institute for Reproductive Medicine. This study was approved by the Institutional Review Board of Eastern Virginia Medical School and written informed consent was obtained from all participants. After liquefaction, the semen sample was washed with mHTF (Irvine Scientific, Santa Ana, CA, USA) supplemented with $0.3 \%$ BSA (Sigma, St. Louis, MO, USA). The spermatozoa were washed twice with mHTFBSA and centrifuged for $10 \mathrm{~min}$ at $300 \mathrm{~g}$. After the second wash, the supernatant was removed and the pellet was resuspended in fresh mHTF-BSA. Next, washed sperm cells were treated with $1 \mathrm{mM}$ MitoTracker green FM (Molecular Probes, Eugene, OR, USA) for $15 \mathrm{~min}$ at $37^{\circ} \mathrm{C}$ for visualization of the sperm tail in subsequent microscopy. After MitoTracker treatment, the plasma membranes from sperm cells were removed using $0.5 \%$ Triton X-100 (TX) (Sigma) prepared in PBS (Sigma) to generate AD sperm. The cell suspension was vortexed for $1 \mathrm{~min}$ then sperm cells were washed in mHTF-BSA three more times prior to being used for ICSI. Washed sperm without TX treatment were used as the control-AI sperm.

\section{Evaluation of acrosomal membrane status}

Acrosomal membrane staining was performed according to the procedure previously described [28] with some modifi-
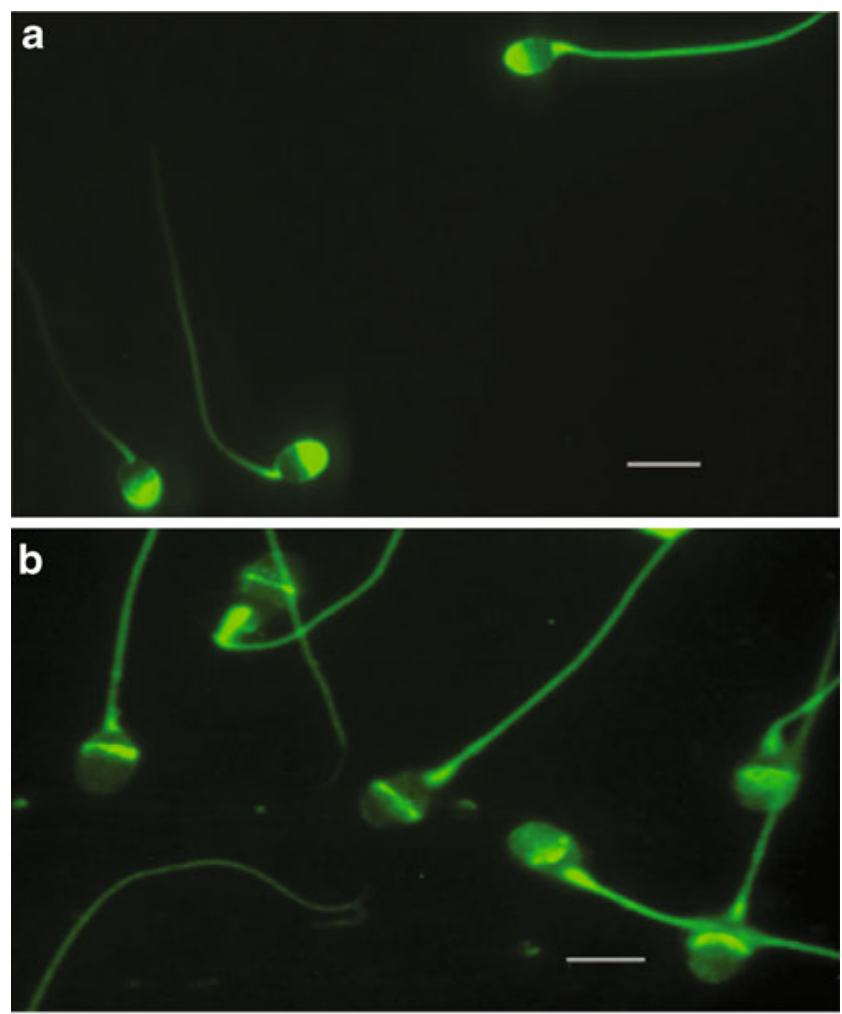

Fig. 1 Acrosomal status of human sperm as revealed by PNA staining (FITC-PNA: green). a Acrosome intact (AI) sperm, note intense fluorescence of the acrosomal cap. b Sperm treated with TX showing weak or no fluorescence were determined to be acrosome depleted (AD). Scale bar $=10 \mu \mathrm{m}$ 
cations. In brief, the sperm samples treated with TX and those serving as control samples were smeared onto microscope slides, air dried, and fixed with absolute methanol. Fixed sperm cells were treated with $100 \mu \mathrm{g} / \mathrm{ml}$ FITC-labeled peanut agglutinin (FITC-PNA) (Sigma) in PBS at $37^{\circ} \mathrm{C}$ for $30 \mathrm{~min}$. After washing with PBS, the samples were mounted with Vectashield containing 4', 6'-diamidino2-phenylindole (DAPI) (Vector Laboratories, Burlingame,
CA, USA) and then evaluated using a fluorescence microscope (Leitz Ortholux, Wetzlar, Germany).

Oocyte preparation and ICSI

Frozen hamster oocytes (HO) (Conception Technologies, San Diego, CA, USA) were thawed and washed for $10 \mathrm{~min}$ in mHTF supplemented with 10\% SSS (mHTF-SSS) (Irvine a
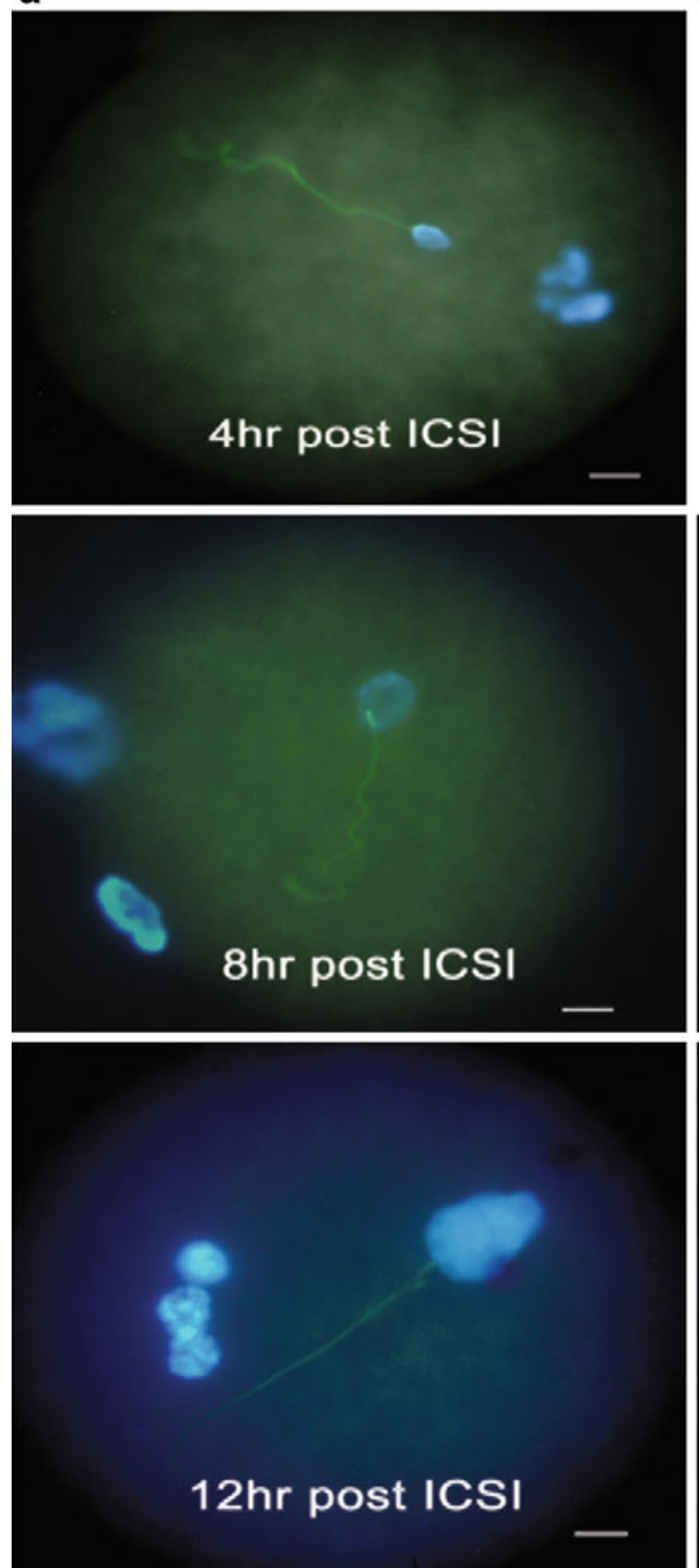

Fig. 2 Transformations of human sperm nuclei in hamster oocytes following heterologous ICSI. Total nuclear DNA stained with DAPI (blue) and sperm tail stained with Mitotracker (green). a Typical images of nuclear decondensation of AD sperm cells. b Mean nuclear b

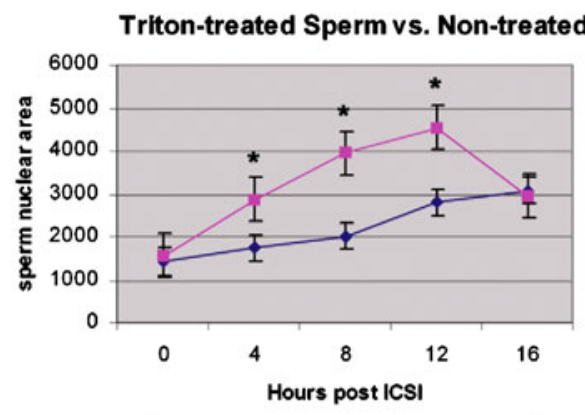

C
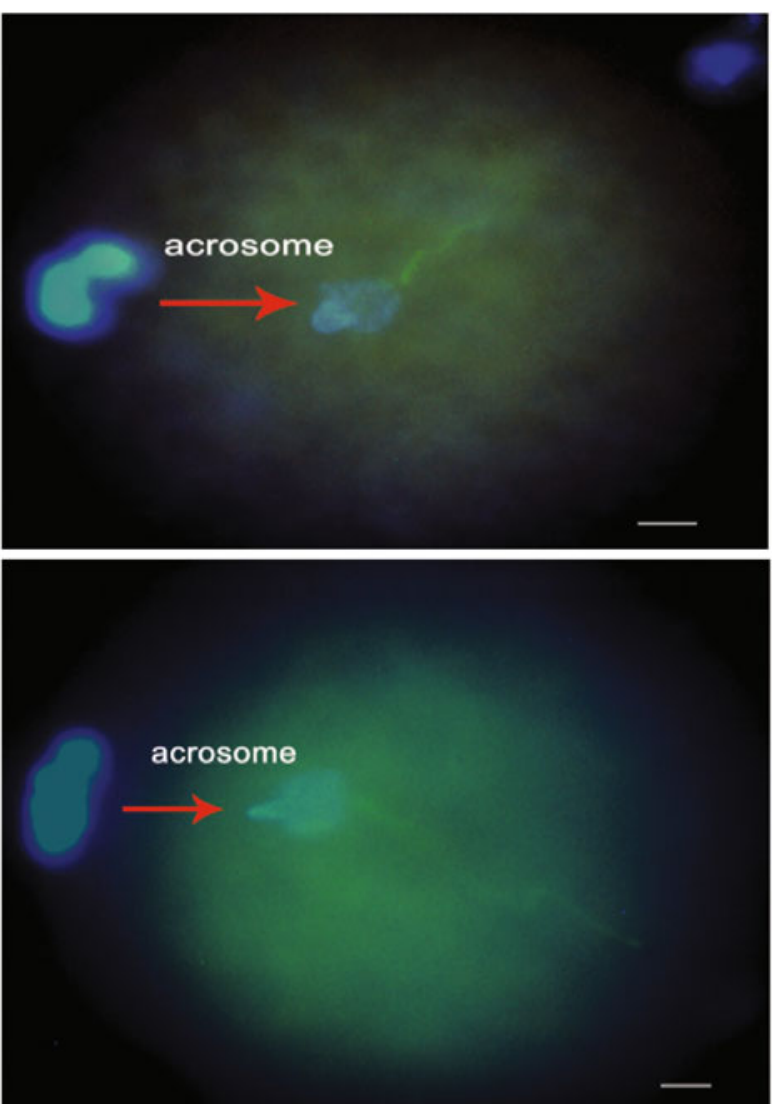

area for TX treated and control spermatozoa. Y-error bars indicate S. E.M.; * indicates significant difference $(p<0.0001)$. c mPN formation with retention of the acrosome in AI sperm often results in abnormal nuclear decondensation. Scale bar $=10 \mu \mathrm{m}$ 
Scientific, Santa Ana, CA, USA) at room temperature before being placed into P1 medium (Irvine Scientific) supplemented with $10 \%$ SSS. Viable oocytes $(97 \%)$ were incubated at $37^{\circ} \mathrm{C}$ in $5 \% \mathrm{CO}_{2}$ prior the ICSI procedure.

ICSI was carried out as previously described [29]. Briefly, $1 \mu \mathrm{l}$ of sperm suspension was diluted with $4 \mu \mathrm{l}$ of $10 \%$ polyvinyl pyrolidone (Irvine Scientific) and placed in the center of an injection dish. Each frozenthawed mature (metaphase II) HO was placed in $5 \mu \mathrm{l}$ of mHTF-SSS surrounding the central drop containing the sperm suspension and covered with mineral oil. At least 50 metaphase II HO were microinjected for each experimental time (total $n=502$ injected oocytes) using Narishige micromanipulators (Narishige, Tokyo, Japan) mounted on a phase-contrast inverted microscope (Olympus, Tokyo, Japan) at $400 \times$ magnification. The selected spermatozoon was aspirated into the injecting micropipette and introduced through the zona pellucida into the ooplasm. The micropipette was then slowly withdrawn and the injected oocytes were kept at $37^{\circ} \mathrm{C}$ in $5 \% \mathrm{CO}_{2}$ for $4,8,12$, and $16 \mathrm{~h}$ in $\mathrm{P} 1$ medium with $10 \% \mathrm{SSS}$. After the respective incubation times, degenerate zygotes (swollen or dark) were discarded and viable oocytes were immediately fixed with $2 \%$ paraformaldehyde (Sigma) and analyzed for sperm nuclear area.

\section{Microscopy and data analysis}

HO were mounted using Vectashield containing DAPI. Total nuclear DNA was visualized on a fluorescence microscope (Leitz Ortholux, Wetzlar, Germany) using selective or triple-band pass filters and the oil immersion $60 \times 1.4$ numerical aperture objective. Images were collected using a MagnaFire digital color camera and MicroFire software (Optronics, Goleta, CA, USA) and processed using Image $\mathrm{J}$ software (National Institute of Mental Health, Bethesda, Maryland, USA). Approximately 50 nuclei were observed for each time point post ICSI for both groups. Sperm samples from the TX treated and control groups were also analyzed for nuclear area size (0 h reference point).

Male pronuclei decondensation was evaluated after staining with DAPI. The surface area of $\mathrm{mPN}$ was measured using Image J. Areas of sperm $/ \mathrm{mPN}$ were evaluated on the analyzer screen. After interactive demarcation of the circumference of the nuclei, the surface areas were automatically calculated by utilizing the enclosed pixels areas. Statistical analysis of nuclear size was conducted using Microsoft Office Excel 2003 (Microsoft, Bellevue, WA, USA). Data were compared by Chi-square and Fisher's exact $t$-test using the GraphPad InStat software (GraphPad Software, Inc., La Jolla, CA, USA).

\section{Results}

Two categories of sperm were used for ICSI: AI (control) and $\mathrm{AD}$ sperm. To obtain $\mathrm{AD}$ sperm acrosome components were removed by Triton X-100 (TX). To evaluate acrosome status we used sperm staining with fluorescent labeled peanut agglutinin (PNA). AI sperm displayed intense fluorescence of the acrosomal cap resulting from PNA binding indicating an intact outer membrane (Fig. 1a). In AD sperm, no fluorescence in the acrosome area was observed indicating partial or complete loss of the outer membrane (Fig. 1b). Numerical evaluation of sperm samples demonstrated that treatment with TX generated $95.5 \%$ of $\mathrm{AD}$ cells. In the control sperm, $12 \%$ of cells were lacking acrosome due to damage acquired during sperm processing. The difference between the groups was statistically significant $(p<0.0001)$.

Heterologous ICSI resulted in production of $82.9 \%$ viable zygotes. Male pronuclear decondensation (mPN were identified by tail staining with Mitotracker) was observed in $60.2 \%$ of the viable oocytes.

Following ICSI into HO, human sperm decondensed in a time-dependent manner as illustrated by DNA staining with DAPI (Fig. 2a). Progressive mPN swelling was observed in both the $\mathrm{AD}$ and $\mathrm{AI}$ groups. In Fig. 2b, human sperm treated with TX were shown to decondense more rapidly and to a greater extent than the control sperm at 4,8 and $12 \mathrm{~h}$ post ICSI $(p<0.0001)$. Pronuclear area gradually increased with swelling reaching a maximum at $12 \mathrm{~h}$ for AD sperm. Thereafter, the nuclei shrunk to dimensions similar to the $4 \mathrm{~h}$ time point. At $12 \mathrm{~h}$ post ICSI, the average area of $\mathrm{AD} \mathrm{mPN}$ was three times greater than in sperm. Maximal value of AI mPN was only twice greater than in sperm. Table 1 shows the mean nuclear area for each group and time point post ICSI.

Some AI mPN demonstrated uneven nuclei decondensation, retention of the acrosome resulted in more condensed DNA in subacrosomal part of PN (Fig. 2c).

The frequency distribution of mean nuclear area for $\mathrm{AD}$ sperm is demonstrated in Fig. 3. Noticeably for any given time point post ICSI, we observed heterogeneity in $\mathrm{MPN}$ size. This was true for both $\mathrm{AD}$ (Fig. 3) and $\mathrm{AI}$ (data not shown) sperm.

Table $1 \mathrm{mPN}$ area of AD (TX treated) and AI (control) sperm at different time points post heterologous ICSI

\begin{tabular}{lllr}
\hline Time & Triton-treated & Control & $p$ value \\
\hline $0 \mathrm{~h}$ & $1,559 \pm 26$ & $1,430 \pm 39$ & $\mathrm{NS}$ \\
$4 \mathrm{~h}$ & $2,868 \pm 224$ & $1,748 \pm 233$ & $(p<0.0001)$ \\
$8 \mathrm{~h}$ & $3,942 \pm 517$ & $2,017 \pm 170$ & $(p<0.0001)$ \\
$12 \mathrm{~h}$ & $4,540 \pm 387$ & $2,808 \pm 257$ & $(p<0.0001)$ \\
$16 \mathrm{~h}$ & $2,651 \pm 475$ & $3,074 \pm 328$ & $\mathrm{NS}$ \\
\hline
\end{tabular}

Values are mean \pm SEM 

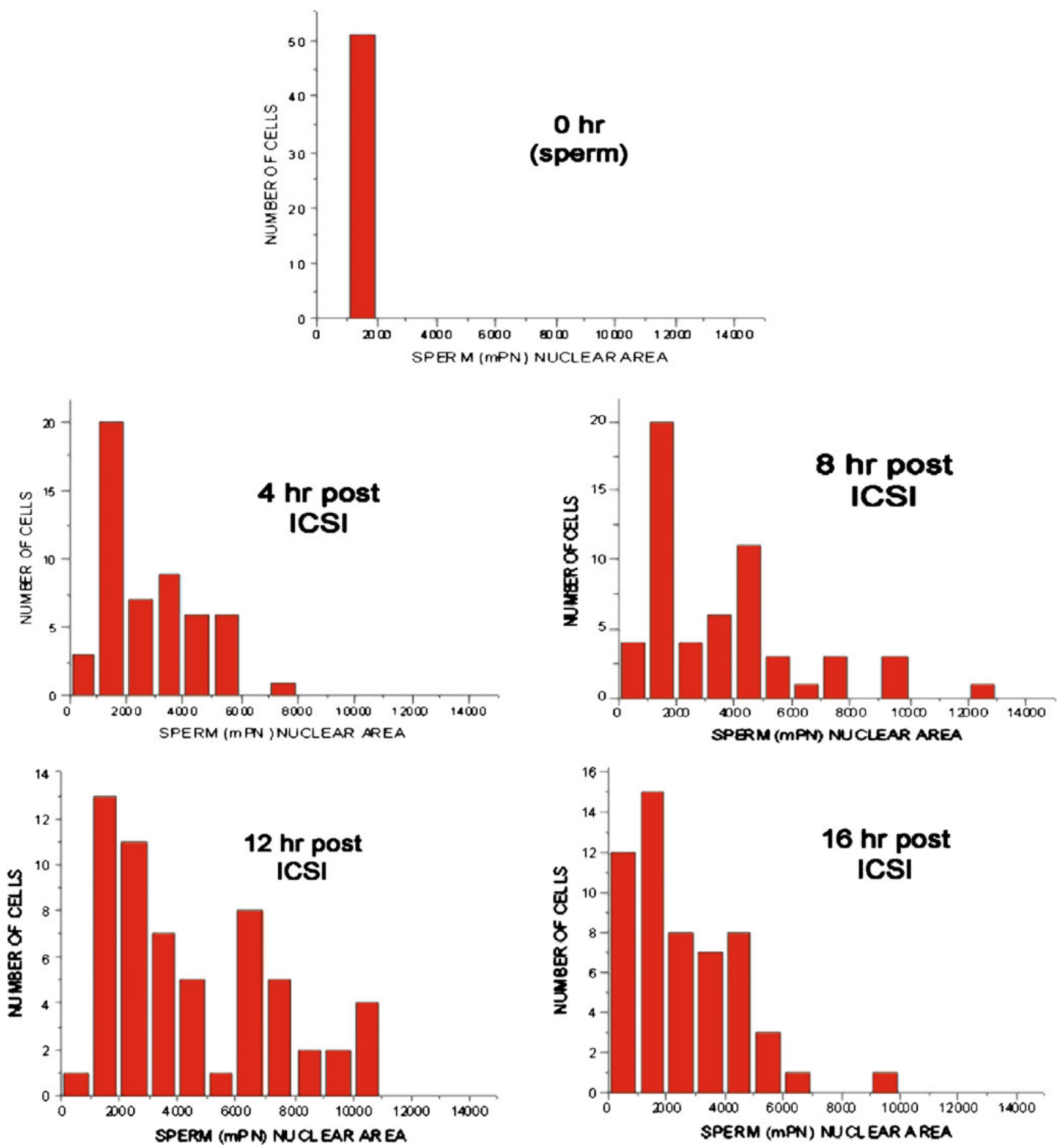

Fig. 3 Frequency distribution of mean $\mathrm{mPN}$ area (pixels/cm) demonstrates heterogeneity of sperm population (AD sperm cells)

\section{Discussion}

The ability of spermatozoa to fertilize oocytes depends on a sequence of events ending ultimately in the remodeling of the sperm chromatin from supercompact state packed by protamins to a relaxed nucleosomal structure. The present study examined human sperm nuclei following ICSI into
HO. Four hours post ICSI, developing human mPN was already more decondensed than "native" sperm $(0 \mathrm{~h}$ reference point). Decondensation of human mPN progressed in a time-dependent manner in both the $\mathrm{AD}$ and $\mathrm{AI}$ groups; however, $\mathrm{mPN}$ resulting from injection of $\mathrm{AD}$ sperm were shown to reach a maximum mean area at $12 \mathrm{~h}$ post injection after which time they shrunk to dimensions 
similar to $4 \mathrm{~h}$ post ICSI (Fig. 2b). During natural fertilization, after entry into the ooplasm, sperm chromatin initially becomes dispersed and then transiently recondenses into a smaller mass [3]. Apparently, this feature was reflected in the current heterologous ICSI approach. Similar decondensation/recondensation was observed in our previous study using the incubation of human sperm in Xenopus egg extract [30].

Within a given sample of spermatozoa, variations in mPN development as judged by nuclear area size were observed (Fig. 3). This may reflect the intrinsic heterogeneity in the mature human sperm population. One of the possible reasons for such variation may be due to the sperm-specific histone TSH2B $[31,32]$. This histone is present in only a $30 \%$ subpopulation of sperm cells. Significant correlation between TSH2B level and CMA3 staining of individual spermatozoa resulting from loosened packaging of DNA has been demonstrated [33]. In studies using the Xenopus egg extract model, sperm cells containing TSH2B decondensed more rapidly and to a greater extent than did spermatozoa without this histone [34]. Other possible reasons for size heterogeneity of mPN may include sperm DNA damage, cell to cell variability in sperm DNA methylation, or heterogeneity of oocytes.

One of the major differences between natural fertilization and ICSI is the absence of sperm modification which naturally occurs during sperm entry at cumulus cells, zona pellucida and oolemna [35]. Typically acrosome intact sperm are used for the ICSI procedure. In the present study both $\mathrm{AI}$ and $\mathrm{AD}$ sperm were used. In $\mathrm{AD}$ sperm, the perninuclear theca was completely removed (Fig. 1b). Importantly, sperm treated with the non-ionic detergent TX, used for solubilizing membrane proteins, developed $\mathrm{mPN}$ at a more advanced rate with larger mean nuclear area than non treated sperm (Fig. 2b). Similarly,in homologous rat ICSI, the timing of pronuclear formation using TX or lysolecithin treated sperm was significantly accelerated compared to control sperm [25]. In the mouse, homologous ICSI of TX treated sperm yielded no difference in fertilization, blastocyst, implantation and live birth rates compared to control sperm [36]. Also, in the heterologous mouse ICSI model using TX treated human sperm, sperm nuclei were examined in the oocytes at various time points post injection and no significant damage was reported [37]. These reports demonstrate the safety of TX use for permeabilization of sperm for ICSI.

During standard ICSI procedure, in addition to overall delay in $\mathrm{mPN}$ development and as proposed paternal genome activation, individual sperm chromosomes might be differentially affected. Indeed, in human sperm, chromosomes occupy defined preferred intranuclear positions $[7,10,38]$. Paternal genome is activated early after fertilization, during $\mathrm{S} / \mathrm{G} 2$ phase of the first cell cycle i.e. before syngamy [39]. Therefore, specific chromosome positioning in sperm may determine their non-random and ordered activation [3, 40, 41]. An increased rate of sex chromosomal anomalies has been reported in human babies conceived by ICSI [42]. This increase could be due to the fact that the sex chromosome $\mathrm{X}$ was found to be preferentially localized at the subacrosomal region of the sperm $[8,10,43]$. It may be suggested that these anomalies, at least in part, are connected with delayed chromosome processing of sperm chromosomes residing in the apical tip [43]. Furthermore, preservation of intact acrosome during ICSI most probably is the major cause of delayed or hindered decondensation of the apical region of the sperm during PN development (see example Fig. 2c).

In conclusion, we demonstrated the kinetics of human sperm nuclei transformation under the influence of ooplasm in a heterologous ICSI model and showed the role of acrosome preservation on sperm DNA decondensation. We propose that human-hamster heterologous ICSI may mimic major events that occur during natural fertilization in humans. However, one limitation to this approach is that in the hamster the centrosome is maternally inherited while it is paternally inherited in the human. To our knowledge, little information is available regarding the role of human sperm centrosomal function for mPN decondesation and chromatin remodeling. Due to the impossibility to use homologous human ICSI for research, the heterologous ICSI approach as described here may be an effective approach to study processing of individual sperm chromosomes/chromosomal domains during $\mathrm{mPN}$ development. Experiments in this direction are currently underway in our laboratory.

\section{References}

1. Collas P, Poccia D. Remodeling the sperm nucleus into a male pronucleus at fertilization. Theriogenology. 1998;49:67-81.

2. Sutovsky P, Schatten G. Paternal contributions to the mammalian zygote: fertilization after sperm-egg fusion. Int Rev Cytol. 2000;195:1-65.

3. McLay DW, Clarke HJ. Remodeling the paternal chromatin at fertilization in mammals. Reproduction. 2003;125:625-33.

4. Fulka H, Mrazek M, Tepla O, Fulka Jr J. DNA methylation pattern in human zygotes and developing embryos. Reproduction. 2004;128:703-8.

5. Zalensky AO, Allen MJ, Kobayashi A, Zalenskaya IA, Balhorn R, Bradbury EM. Well-defined genome architecture in the human sperm nucleus. Chromosoma. 1995; 103:577-90.

6. Haaf T, Ward DC. Higher order nuclear structure in mammalian sperm revealed by in situ hybridization and extended chromatin fibers. Exp Cell Res. 1995;219:604-11.

7. Mudrak O, Tomilin N, Zalensky A. Chromosome architecture in the decondensing human sperm nucleus. J Cell Sci. 2005;118:4541-50.

8. Hazzouri M, Rousseaux S, Mongelard F, Usson Y, Pelletier R, Faure AK, et al. Genome organization in the human sperm 
nucleus studied by FISH and confocal microscopy. Mol Reprod Dev. 2000;55:307-15.

9. Tilgen N, Guttenbach M, Schmid M. Heterochromatin is not an adequate explanation for close proximity of interphase chromosomes 1-Y, 9-Y, and 16-Y in human spermatozoa. Exp Cell Res. 2001;265:283-7.

10. Zalenskaya IA, Zalensky AO. Non-Random positioning of chromosomes in human sperm nuclei. Chromosome Res. 2004;12:1-11.

11. Fulka H, Barnetova I, Mosko T, Fulka J. Epigenetic analysis of human spermatozoa after injection into ovulated mouse oocytes. Hum Reprod. 2008;23:627-34.

12. Yanagimachi R, Yanagimachi H, Rogers BJ. The use of zona free animal ova as a test system for the assessment of the fertilizing capacity of human spermatozoa. Biol Reprod. 1976;15:471-6.

13. Rogers BJ, Perreault SD, Brentwood BJ, McCarville C, Hale R, Soderdahl DW. Variability in human-hamster in vitro assay for fertility evaluation. Fertil Steril. 1983;39:204-11.

14. Aiken RJ. Diagnostic value of the hamster oocyte penetration assay. Int J Androl. 1984;7:273-5.

15. Hewitson L, Haavisto L, Simerly C, Jones J, Schatten G. Microtubule organization and chromatin configurations in hamster oocytes during fertilization and parthenogenetic activation, and after insemination with human sperm. Biol Reprod. 1997;57:96775 .

16. Rosenbusch BE. Cytogenetics of human spermatozoa: what about the reproductive relevance of structural chromosome aberrations? J Assist Reprod Genet. 1995;12:375-83.

17. Yanagida K, Yanagimachi R, Perreault SD, Kleinfeld RG. Thermostability of sperm nuclei assessed by microinjection into hamster oocytes. Biol Reprod. 1991;44:440-7.

18. Araki Y, Yoshizawa M, Araki Y. A novel method for chromosome analysis of human sperm using enucleated mouse oocytes. Hum Reprod. 2005;20:1244-7.

19. Ajduk A, Yamauchi Y, Ward MA. Sperm chromatin remodeling after intracytoplasmic sperm injection differs from that of in vitro fertilization. Biol Reprod. 2006;75:442-51.

20. Sutovsky P, Hewisotn L, Simerly CR, Tengowski MW, Navara CS, Hasvisto A, et al. Intracytoplasmic sperm injection for Rhesus monkey fertilization results in unusual chromatin, cytoskeletal, and membrane events, but eventually leads to pronuclear development and sperm aster assembly. Hum Reprod. 1996;11:1703-12.

21. Terada Y, Luetjens CM, Sutovsky P, Schatten G. Atypical decondensation of the sperm nucleus, delayed replication of the male genome, and sex chromosome positioning following intracytoplasmic human sperm injection (ICSI) into golden hamster eggs: does ICSI itself introduce chromosomal anomalies? Fertil Steril. 2000;74:454-60.

22. Katayama M, Koshida M, Miyake M. Fate of the acrosome in ooplasm in pigs after IVF and ICSI. Hum Reprod. 2002;17:265764.

23. Yanagimachi R. Intracytoplasmic injection of spermatozoa and spermatogenic cells: its biology and applications in human and animals. Reprod Biomed Online. 2005;10:247-88.

24. Van Steirteghem A, Bonduelle M, Devroey P. Liebaers: follow-up of children born after ICSI. Hum Reprod. 2002;8:111-6.

25. Seita Y, Junya ITO, Kashiwazaki N. Removal of acrosomal membrane from sperm head improves development of rat zygotes derived from intracytoplasmic sperm injection. J Reprod Dev 2009; (in press).

26. Morozumi K, Yanagimachi R. Incorporation of the acrosome into the oocyte during intracytoplasmic sperm injection could be potentially hazardous to embryo development. Proc Natl Acad Sci. 2005;102:14209-14.

27. World Health Organization (WHO). Laboratory manual for the examination of human semen and sperm-cervical mucus interaction. 3rd ed. Cambridge: Cambridge University Press; 1992.

28. Fazeli A, Hage WJ, Cheng FP, Voorhout WF, Mark SA, Bevers $\mathrm{MM}$, et al. Acrosome-intact boar spermatozoa initiate binding to the homologous zona pellucida in vitro. Biol Reprod. 1997;56: 430-8.

29. Palermo G, Joris H, Devroey P, Van Steirteghem AC. Pregnancies after intracytoplasmic injection of single spermatozoon into an oocyte. Lancet. 1992;340:17-8.

30. Mudrak O, Chandra R, Jones E, Godfrey E, Zalensky A. Reorganization of human sperm nuclear architecture during formation of pronuclei in a model system. Reprod Fertil Dev. 2009;21:665-71.

31. Van Roijen JH, Ooms MP, Spaargaren MC, Baarends WM, Weber RFA, Grootegoed JA, et al. Immunoexpression of testis-specific histone $2 \mathrm{~B}$ in human spermatozoa and testis tissue. Hum Reprod. 1998;13:1559-66.

32. Zalensky AO, Siino JS, Gineitis AA, Zalenskaya IA, Tomilin NV, Yau P, et al. Human testis/sperm specific histone H2B (hTSH2B); Molecular cloning and characterization. J Biol Chem. 2002;277: 43474-80.

33. Singleton S, Mudrak O, Morshedi M, Oehninger S, Zalenskaya I, Zalensky A. Characterization of a human sperm cell subpopulation marked by the presence of the TSH2B histone. Reprod Fertil Dev. 2007;19:392-7.

34. Singleton S, Zalensky A, Doncel GF, Morshedi M, Zalenskaya IA. Testis/sperm-specific histone $2 \mathrm{~B}$ in the sperm donors and subfertile patients: variability and relation to chromatin packaging. Hum Reprod. 2007;22:743-50.

35. Yanagimachi R. Mammalian fertilization. In: Knobil E, Neill JD, editors. The physiology of reproduction. 2nd ed. New York: Raven; 1994. p. 189-317.

36. Ahmadi A, Ng SC. Fertilization and development of mouse oocytes injected with membrane-damaged spermatozoa. Hum Reprod. 1997;12:2797-801.

37. Kasai T, Hoshi K, Yanagimachi R. Effect of sperm immobilization and demembranation on the oocyte activation rate in the mouse. Zygote. 1999;7:187-93.

38. Manvelyan M, Hunstig F, Bhatt S, Mrasek K, Pellestor F, Weise A, et al. Chromosome distribution in human sperm-a 3D multicolor banding-study. Mol Cytogenet. 2008;14:1-25.

39. Adenot PG, Mercier Y, Renard JP, Thompson EM. Differential H4 acetylation of paternal and maternal chromatin precedes DNA replication and differential transcriptional activity in pronuclei of 1-cell mouse embryos. Development. 1997;124:4615-25.

40. Schultz RM, Worrad DM. Role of chromatin structure in zygotic gene activation in the mammalian embryo. Semin Cell Biol. 1995;6:201-8.

41. Zalensky A, Zalenskaya I. Organization of chromosomes in spermatozoa: an additional layer of epigenetic information? Biochem Soc Trans. 2007;35:609-11.

42. Bonduelle M, Liebaers I, Derde MP, Camus M, Devroey P, Van Steirtegham A. Neonatal data on a cohort of 2889 infants born after ICSI (1991-1999) and of 2995 infants born after IVF (19831999). Hum Reprod. 2002;17:671-94.

43. Luetjens CM, Payne C, Schatten G. Non-random chromosome positioning in human sperm and sex chromosome anomalies following intracytoplasmic sperm injection. Lancet. 1999;353:1240. 